\title{
EFFECTIVENESS AND SAFETY OF THE SEQUENTIAL USE OF A SECOND AND THIRD ANTI-TNF AGENT IN PATIENTS WITH INFLAMMATORY BOWEL DISEASE: RESULTS FROM THE ENEIDA REGISTRY
}

SHORT TITLE: Sequential use of anti-TNFs in IBD patients

\section{AUTHORS}

María José Casanova ${ }^{1}$, María Chaparro ${ }^{1}$, Miguel Mínguez ${ }^{2}$, Elena Ricart ${ }^{3}$, Carlos Taxonera ${ }^{4}$, Santiago García-López ${ }^{5}$, Jordi Guardiola ${ }^{6}$, Antonio López-San Román ${ }^{7}$, Eva Iglesias $^{8}$, Belén Beltrán ${ }^{9}$, Beatriz Sicilia ${ }^{10}$, María Isabel Vera ${ }^{11}$, Joaquín Hinojosa ${ }^{12}$, Sabino Riestra $^{13}$, Eugeni Domènech ${ }^{14}$, Xavier Calvet ${ }^{15}$, José Lázaro Pérez-Calle ${ }^{16}$, María Dolores Martín-Arranz $^{17}$, Xavier Aldeguer ${ }^{18}$, Montserrat Rivero ${ }^{19}$, David Monfort ${ }^{20}$, Jesús Barrio ${ }^{21}$, María Esteve $^{22}$, Lucía Márquez ${ }^{23}$, Rufo Lorente ${ }^{24}$, Esther García-Planella ${ }^{25}$, Luisa de Castro ${ }^{26}$, Fernando Bermejo ${ }^{27}$, Olga Merino ${ }^{28}$, Antonio Rodríguez-Pérez ${ }^{29}$, Pilar Martínez-Montiel ${ }^{30}$, Manuel Van Domselaar ${ }^{31}$, Guillermo Alcaín ${ }^{32}$, Manuel Domínguez-Cajal ${ }^{33}$, Carmen Muñoz ${ }^{34}$, Fernando Gomollón ${ }^{35}$, Luis Fernández-Salazar ${ }^{36}$; Mariana Fe García-Sepulcre ${ }^{37}$, Iago Rodríguez-Lago $^{38}$, Ana Gutiérrez ${ }^{39}$, Federico Argüelles-Arias ${ }^{40}$, Cristina Rodriguez ${ }^{41}$, Gloria Esther Rodríguez ${ }^{42}$, Luis Bujanda ${ }^{43}$, Jordina Llaó ${ }^{44}$, Pilar Varela ${ }^{45}$, Laura Ramos ${ }^{46}$, José María Huguet $^{47}$, Pedro Almela ${ }^{48}$, Patricia Romero ${ }^{49}$, Mercè Navarro-Llavat ${ }^{50}$, Águeda Abad ${ }^{51}$, Patricia Ramírez-de la Piscina ${ }^{52}$, Alfredo J. Lucendo ${ }^{53}$, Eva Sesé ${ }^{54}$, Rosa Eva Madrigal ${ }^{55}$, Mara Charro ${ }^{56}$, Antonio García-Herola ${ }^{57}$, Ramón Pajares ${ }^{58}$, Sam Khorrami ${ }^{59}$ and Javier P. Gisbert ${ }^{1}$.

Gastroenterology Departments from: ${ }^{1}$ Hospital Universitario de La Princesa, Instituto de Investigación Sanitaria Princesa (IIS-IP), Universidad Autónoma de Madrid, and Centro de Investigación Biomédica en Red de Enfermedades Hepáticas y Digestivas (CIBEREHD), Madrid; ${ }^{2}$ Hospital Clínico Valencia, Valencia; ${ }^{3}$ Hospital Clínic i Provincial, Barcelona, CIBEREHD and IDIBAPS; ${ }^{4}$ Hospital Universitario Clínico San Carlos, Madrid; ${ }^{5}$ Hospital Universitario Miguel Servet, Zaragoza, and CIBEREHD; ${ }^{6}$ Hospital Universitario de Bellvitge, Barcelona; ${ }^{7}$ Hospital Universitario Ramón y Cajal, Madrid; ${ }^{8}$ Hospital Universitario Reina Sofía, Córdoba; ${ }^{9}$ Hospital Universitario La Fe, Valencia, and CIBEREHD; ${ }^{10}$ Hospital Universitario de Burgos, Burgos; ${ }^{11}$ Hospital Universitario Puerta de Hierro Majadahonda, Madrid; ${ }^{12}$ Hospital de Manises, Valencia; ${ }^{13}$ Hospital Universitario Central de Asturias, Oviedo; ${ }^{14}$ Hospital Universitario Germans Trias i Pujol, Badalona, and CIBEREHD, ${ }^{15}$ Hospital de Sabadell. Corporació Sanitària Universitària Parc Taulí, Sabadell, and CIBEREHD; ${ }^{16}$ Hospital Universitario Fundación de Alcorcón, Madrid; ${ }^{17}$ Hospital Universitario La Paz, Madrid and Instituto de Investigación de La Paz (IdiPaz); ${ }^{18}$ Hospital Universitari de Girona Dr. Josep Trueta, Girona; ${ }^{19}$ Hospital Universitario Marqués de Valdecilla, Santander and IDIVAL; ${ }^{20}$ Consorci Sanitari Terrassa, Terrassa; ${ }^{21}$ Hospital Universitario Río Hortega, Valladolid; ${ }^{22}$ Hospital Universitario Mútua Terrassa, Terrassa, and CIBEREHD; ${ }^{23}$ Hospital del Mar, Barcelona; ${ }^{24}$ Hospital General Universitario de Ciudad Real, Ciudad Real; ${ }^{25}$ Hospital de la Santa Creu i Sant Pau, Barcelona; ${ }^{26}$ Complejo Hospitalario Universitario de Vigo, Vigo; ${ }^{27}$ Hospital Universitario de Fuenlabrada and Instituto de Investigación de La Paz (IdiPaz), Madrid; ${ }^{28}$ Hospital Universitario Cruces, Barakaldo; 
${ }^{29}$ Hospital Clínico Universitario de Salamanca, Salamanca; ${ }^{30}$ Hospital Universitario Doce de Octubre, Madrid; ${ }^{31}$ Hospital de Torrejón, Madrid; ${ }^{32}$ Hospital Universitario Virgen de la Victoria, Málaga; ${ }^{33}$ Hospital General San Jorge, Huesca; ${ }^{34}$ Hospital de Basurto, Bilbao; ${ }^{35}$ Hospital Clínico Universitario Lozano Blesa, Zaragoza; ${ }^{36}$ Hospital Clínico Universitario de Valladolid, Valladolid; ${ }^{37}$ Hospital General Universitario de Elche, Alicante; ${ }^{38}$ Hospital de Galdakao-Usansolo, Galdakao; ${ }^{39}$ Hospital General Universitario de Alicante, Alicante, and CIBEREHD; ${ }^{40}$ Hospital Universitario Virgen Macarena, Sevilla; ${ }^{41}$ Complejo Hospitalario de Navarra, Instituto de Investigación Sanitaria de Navarra (IdiSNA), Pamplona; ${ }^{42}$ Hospital Universitario Nuestra Señora de la Candelaria, Santa Cruz de Tenerife; ${ }^{43}$ Hospital Universitario de Donostia, Instituto Biodonostia, Universidad del País Vasco (UPV/EHU), Donostia, and CIBEREHD; ${ }^{44}$ ALTHAIA Xarxa Assistencial Universitària de Manresa, Manresa; ${ }^{45}$ Hospital Universitario de Cabueñes, Gijón; ${ }^{46}$ Hospital Universitario de Canarias, La Laguna; ${ }^{47}$ Consorcio Hospital General Universitario de Valencia, Valencia; ${ }^{48}$ Hospital General Universitario de Castellón, Castellón; ${ }^{49}$ Hospital General Universitario de Santa Lucía, Murcia; ${ }^{50}$ Hospital de Sant Joan Despí Moisès Broggi, Sant Joan Despí; ${ }^{51}$ Hospital de Viladecans, Barcelona; ${ }^{52}$ Hospital Universitario de Álava, Vitoria; ${ }^{53}$ Hospital General de Tomelloso; Ciudad Real; ${ }^{54}$ Hospital Universitario Arnau de Vilanova, Lleida; ${ }^{55}$ Complejo Asistencial Universitario de Palencia, Palencia; ${ }^{56}$ Hospital Royo Villanova, Zaragoza; ${ }^{57}$ Hospital Marina Baixa, Alicante; ${ }^{58}$ Hospital Universitario Infanta Sofía, Madrid; ${ }^{59}$ Hospital Universitario Son Espases, Palma de Mallorca. Spain.

\section{CORRESPONDENCE}

Javier P. Gisbert, M.D.

Gastroenterology Unit.

Hospital Universitario de La Princesa

Diego de León, 62. 28006 Madrid. Spain

Tel.: 34-913093911; Fax: 34-915204013.

E-mail: javier.p.gisbert@gmail.com

ACKNOWLEDGEMENTS: The authors thank the Spanish Working Group on Crohn's Disease and Ulcerative Colitis (GETECCU) for promoting the ENEIDA registry and the support from Pfizer, Abbvie, Takeda, Kern and MSD. Financial support: none

REQUIRED SUMMARY: Almost half of the 1,122 patients who switched to a second anti-TNF after primary failure, secondary failure or intolerance to the first anti-TNF achieved remission after switching. Factors associated with loss of response were type of IBD and combo therapy. 
BACKGROUND: The effectiveness of the switch to another anti-TNF agent is barely known.

AIMS: To analyse the effectiveness and safety of the treatment with a second and a third antiTNF drug after intolerance or failure to a previous anti-TNF agent in inflammatory bowel disease (IBD) patients.

METHODS: We included patients diagnosed with IBD from ENEIDA registry who received another anti-TNF after intolerance or failure to a prior anti-TNF agent.

RESULTS: 1,122 patients were included. In the short-term, remission was achieved in 55\% of them with the second anti-TNF. The incidence of loss of response was $19 \%$ per patientyear with the second anti-TNF. Combination therapy $(\mathrm{HR}=2.4 ; 95 \% \mathrm{CI}=1.8-3, \mathrm{P}<0.0001)$ and ulcerative colitis vs. Crohn's disease $(\mathrm{HR}=1.6 ; 95 \% \mathrm{CI}=1.1-2.1, \mathrm{P}=0.005)$ were associated with a higher probability of loss of response. Fifteen percent of the patients had adverse events and $10 \%$ had to discontinue the second anti-TNF. Of the 71 patients who received a third anti-TNF, 55\% achieved remission. The incidence of loss of response was $22 \%$ per patient-year with a third anti-TNF. Adverse events occurred in 7 patients (11\%), but only 1 stopped the drug.

CONCLUSION: Approximately half of the patients who received a second anti-TNF achieved remission; nevertheless, a significant proportion of them subsequently lost response. Combination therapy and type of IBD were associated with loss of response. Remission was achieved in almost $50 \%$ of patients who received a third anti-TNF; nevertheless, a significant proportion of them subsequently lost response.

\section{KEY WORDS}

Inflammatory bowel disease, Crohn's disease, ulcerative colitis, anti-TNF, switch. 


\section{INTRODUCTION}

Anti-TNF drugs are certainly effective in patients diagnosed with inflammatory bowel disease (IBD). Nevertheless, approximately $30 \%$ of IBD patients treated with anti-TNF agents are non-responders to the therapy, and a significant proportion of those who respond experiences intolerance or loss of response over time ${ }^{1}$. The use of a second anti-TNF is a common practice when a first one has failed ${ }^{2}$. In this context, a significant number of patients will be treated with a second anti-TNF. Nevertheless, the clinical management of patients who experiences loss of response to a first anti-TNF agent is done empirically. A recent metaanalysis found that the efficacy of a second anti-TNF drug in patients diagnosed with Crohn's disease (CD) was clearly dependent on the reason for switching treatment ${ }^{2}$. On the other hand, only few studies have evaluated the efficacy of a second anti-TNF in ulcerative colitis (UC) patients, and the remission rates reported are highly variable $\mathrm{e}^{3-9}$. Moreover, a significant number of patients who have an initial response to a second anti-TNF finally lose the response or became intolerant to the $\operatorname{drug}^{10}$. Furthermore, the use of a third anti-TNF agent when the second one has failed is not unusual; however, available data are scarce. In the last few years, new molecules have been incorporated into the therapeutic armamentarium for IBD patients. However, these treatments are not effective in all patients. For this reason, it is important to get the most out of anti-TNF drugs.

The aims of our study were to evaluate the effectiveness and the safety of the sequential use of a second and a third anti-TNF agent after intolerance to or failure of a previous anti-TNF drug, to identify the predictors of remission with a second anti-TNF, and to investigate which variables are associated with the risk of loss of response to a second anti-TNF. 


\section{METHODS}

\section{Study population}

The study included patients that have been diagnosed with CD or UC from ENEIDA registry who received another anti-TNF after intolerance or intolerance to a prior anti-TNF drug in order to achieve clinical remission. ENEIDA is a prospectively maintained registry of the Spanish Working Group in Crohn's disease and Ulcerative Colitis (GETECCU), which includes patients with IBD. The database prospectively records clinical characteristics of the patients, and the use, effectiveness, and adverse events of immunomodulators and biologic therapy. Physicians from IBD centers that are registered in ENEIDA can voluntarily include the data of their patients in ENEIDA. At the time of data extraction, the registry contained 11,866 patients who were exposed to anti-TNF therapy. ENEIDA registry was approved by Research Ethic Committees in all participating centers. All co-authors had access to the study data and have reviewed and approved the final manuscript.

Patients were excluded from the study if the anti-TNF agent was initiated for treatment of extraintestinal manifestations of IBD or as a preventive therapy of post-operative recurrence in CD patients, if the time between the end of the previous anti-TNF and the onset of the second or third anti-TNF was longer than 6 months, and if the previous anti-TNF was stopped for other reasons than failure or intolerance (e.g. elective decision, pregnancy, etc.).

\section{Data collection}

The data collected included demographic data, age at diagnosis, smoking habit, duration of IBD, location, disease extent, disease behavior, perianal disease, extraintestinal manifestations, history of abdominal surgery due to IBD, concomitant therapy with immunomodulators, type of anti-TNF agent, indications for anti-TNF therapy, reasons for 
discontinuation of the anti-TNF, response to anti-TNF and the need to dose escalation, and the occurrence of adverse events.

\section{Definitions of study variables}

Primary failure: it was considered that the patient had a primary failure if she/he did not achieve remission after having received the induction doses of the anti-TNF (non-responders and partial-responders).

Secondary failure: it was considered that a patient had a secondary failure if she/he achieved remission with the anti-TNF agent and then lost effectiveness over time.

Intolerance to treatment: the patient was considered intolerant to the anti-TNF treatment if he/she had adverse events that led to withdraw the drug.

Sequential use of anti-TNF drugs: if the period of time between the end of the first or second anti-TNF drug (due to intolerance, primary failure or secondary failure) and the onset of the next anti-TNF was less than 6 months.

Loss of efficacy: if the patient achieved remission with the anti-TNF agent and then had symptoms compatible with clinical activity together with radiographic, endoscopic and/or biochemical evidence of disease activity that leaded to a dose escalation or to switch to another anti-TNF.

Dose escalation: if the patient was treated with adalimumab (ADA), a reduction in the interval of administration was defined as a dose escalation. A decrease in the administration interval, an increase in the dose or both were considered dose escalation in patients treated with infliximab (IFX)

Concomitant immunomodulators (IMMs): immunosuppressive treatment was considered concomitant with anti-TNF if the patient had been on IMMs (e.g. azathioprine, mercaptopurine or methotrexate) after starting the anti-TNF therapy for a period greater or 
equal than 6 months.

Smoking: patients were categorized as having smoked or as never having smoked.

Clinical remission: a Harvey-Bradshaw index score $\leq 4$ points was considered remisson for luminal $\mathrm{CD}^{11}$. A partial Mayo score $\leq 2$ points was considered remission for UC patients $^{12}$

Short-term effectiveness: it was defined as remission at week 12 (after induction doses)

Long-term effectiveness: it was defined as the proportion of patients that maintained clinical remission with the anti-TNF along time.

\section{Statistical analysis}

In the descriptive analysis, we used the mean and standard deviation for quantitative variables if they were normally distributed. If they were not, we used the median and interquartile range (IQR). The 95\% confidence intervals (95\% CIs) and the percentage were provided for the categorical variables. The $t$ test for independent samples was used to perform comparisons between means. The $\chi^{2}$ test and the Fisher exact test were used to compare the categorical variables if they were normally distributed. If they were not, the Wilcoxon ranksum test was used. A $P<0.05$ was considered statistically significant.

In the case of the second anti-TNF agent, the factors associated with the short-term efficacy were studied with multivariate analyses (logistic regression). The variables that were statistically significant after performing a univariate analysis were included in the multivariate analysis. Moreover, those variables that could be relevant even if they were not statistically significant were also included.

In the patients that achieved remission in the short-term with a second and or third antiTNF agent, Kaplan-Meier curves were used to estimate the long-term maintenance of remission. Long-rank test was used to evaluate the impact of some variables on long-term 
remission. In the group of patients that achieved remission with the second anti-TNF therapy, predictive factors for loss of efficacy was identified using a Cox regression model. All the variables that reached statistical significance in the univariate analysis and those that were considered clinically relevant were included in the multivariate analysis. The dependent variable was the loss of efficacy to a given anti-TNF.

\section{Ethical considerations}

The study was approved by the respective institutional ethics review boards, and was conducted according to the Declaration of Helsinki and Good Clinical Practice guidelines. 


\section{RESULTS}

\section{Study population}

We included 1,122 IBD (CD or UC) patients that switched to a second anti-TNF after failing (primary or secondary failure) or were intolerant to a first anti-TNF drug and met the inclusion criteria. The main characteristics of the study population are summarized in Table 1.

The median duration of the first anti-TNF therapy before switching to a second anti-TNF was 12 months (IQR 4-26).

Most of the patients (67\%) had received IFX as a first anti-TNF agent. Most of the patients $(65 \%)$ received ADA as a second anti-TNF. Approximately half of the patients (51\%) who switched to a third anti-TNF received certolizumab.

Sixty-two percent of the patients received IMMs with the first anti-TNF, 33\% of them continued this therapy after switching to a second anti-TNF drug, and $23 \%$ with the third. The reasons for discontinuation of the first anti-TNF were: secondary failure (51\%), intolerance $(27 \%)$, and primary failure (non-responders and partial responders, $22 \%$ ). In a subanalysis, the mean time from the IBD diagnosis until the start of the second anti-TNF was shorter in patients on combo therapy with the second anti-TNF than in those on anti-TNF monotherapy ( 85 months vs. 102 months, $P=0.003$ ). Similarly, the mean duration of disease until the start of the first anti-TNF was shorter in patients on combo therapy with the second anti-TNF (68 months vs. 82 months, $\mathrm{P}=0.009$ ).

\section{Second anti-TNF: short-term effectiveness}

After the start of the second anti-TNF drug, the median time of follow-up was 14 months (IQR 5-32). In the short-term, 45\% (500) of the patients achieved remission wit the second anti-TNF agent. Patients who switched to a second anti-TNF due to intolerance to the first 
drug had higher remission rates than those who switched due to secondary failure $(52 \%$ vs. $42 \%, P=0.003)$ or primary failure ( $52 \%$ vs. $39 \%, P=0.003)$. We did not find difference between patients who switched due to primary failure vs. secondary failure (39\% vs. $42 \%$, $P=0.5)$. Remission rate in the short-term was similar in CD patients $(46 \%)$ in comparison with UC patients $(41 \%), P=0.06$. Remission rates were similar among the sequences of the antiTNF administration: adalimumab-infliximab or infliximab-adalimumab (48\% vs. $42 \%$, $P=0.07)$.

In the multivariate analyses (Table 2), combo therapy ( $\mathrm{OR}=0.5,95 \% \mathrm{CI}=0.4-0.8)$, withdrawal of the first anti-TNF due to a primary failure (vs. intolerance; OR $=0.6,95 \%$ $\mathrm{CI}=0.4-0.9)$ and withdrawal of the first anti-TNF due to secondary failure (vs. intolerance) (OR $0.6,95 \% \mathrm{CI}=0.5-0.9)$ were associated with a lower probability of achieving remission with the second anti-TNF. Gender, age at diagnosis, smoking history, type of IBD, extraintestinal manifestations and duration of disease were not associated with the effectiveness of a second anti-TNF.

In a subanalysis to evaluate the variables associated with short-term remission in CD patients who switched to a second anti-TNF, none of these factors (plus the localization and phenotype of the disease, the presence of perianal disease, and previous surgery owe to IBD) was statistically significant.

\section{Second anti-TNF: long-term effectiveness}

In the patients who achieved remission with the second anti-TNF, the median time of follow-up of was 19 months (IQR 8-40). After achieving remission with the second anti-TNF, the cumulative incidence of loss of efficacy was $45 \%(95 \% \mathrm{CI}=41-49 \%): 23 \%$ at 1 year, $38 \%$ at 2 years, $66 \%$ at 3 years, and $62 \%$ at 5 years after switching. The proportion of patients that remained in remission during follow-up is shown in figure 1. The incidence of loss of efficacy 
in patients in remission with the second anti-TNF was $19 \%(95 \% \mathrm{CI}=17-22 \%)$ per patientyear.

The univariate analyses showed that combo therapy ( $74 \%$ combo therapy vs. $36 \%$ antiTNF in monotherapy, $\mathrm{P}<0.0001$ ), and type of IBD ( $45 \% \mathrm{CD}$ vs $57 \% \mathrm{UC}, \mathrm{P}=0.009)$ were the only variables associated with the loss of efficacy to a second anti-TNF (figures 2 and 3). In the multivariate analysis, type of IBD (UC vs. $\mathrm{CD} ; \mathrm{HR}=1.6,95 \% \mathrm{CI}=1.1-2.1, \mathrm{P}=0.005$ ) and combo therapy $(\mathrm{HR}=2.4,95 \% \mathrm{CI}=1.8-3, \mathrm{P}<0.0001)$ were associated with a higher probability of loss of efficacy. Gender, smoking history, age at diagnosis, duration of disease, extraintestinal manifestations and reasons to withdraw the first anti-TNF were not associated with the loss of efficacy.

In $C D$ patients, none of these variables (plus the presence of perianal disease, the localization and phenotype of disease, and previous abdominal surgery owe to IBD) had impact on the loss of efficacy to a second anti-TNF agent.

\section{Second anti-TNF: dose-escalation}

Of the 1,122 patients who were treated with a second anti-TNF agent, $21 \%$ (230 patients) needed dose-escalation of the drug. Of these, $42 \%$ achieved remission. The reasons for doseescalation were: secondary failure (84\%), partial response (14\%) and non-response (2\%). The rates of remission were, according to the reason of dose-escalation: $40 \%$ for secondary failure, $57 \%$ for partial response and $0 \%$ for non-responders. Only $22 \%$ of the patients who achieved remission with the second anti-TNF needed to increase the anti-TNF dose during follow-up. The median time to dose escalation was 15 months (IQR 5-37). The median follow-up time after dose escalation was 13 months (IQR 6-26). After escalating the anti-TNF dose, $70 \%$ of the patients achieved remission again. At 1 year, the vast majority of these patients $(89 \%)$ remain in remission. 


\section{Second anti-TNF: safety}

Fifteen percent $(95 \% \mathrm{CI}=13-17 \%)$ of the patients had adverse events. Of these, $66 \%(95 \%$ $\mathrm{CI}=59-74 \%$ ) required discontinuation of the drug. Therefore, $10 \%$ of all the patients treated with a second anti-TNF discontinued the drug because of adverse events. Infusion reactions and infections were the most frequent adverse events, as is shown in Table 3.

\section{Third anti-TNF}

A third anti-TNF was started in 71 patients. Of these, $45 \%$ discontinued the second antiTNF due to primary failure, $30 \%$ due to secondary failure, and $16 \%$ due to intolerance. In Table 4 are summarized the characteristics of the patients. Most of the patients $(51 \%)$ received certolizumab as a third anti-TNF. Twenty-three percent of patients (16) had received combo therapy.

\section{Third anti-TNF: effectiveness}

In the short-term, $55 \%(95 \% \mathrm{CI}=43-67 \%)$ of the patients achieved remission with the third anti-TNF. Of these, $16 \%$ switched to the third agent due to intolerance of the second drug, $39 \%$ due to secondary failure and $45 \%$ due to primary failure. The median follow-up time after switching to a third agent was 9 months (IQR 4-16). The incidence of loss of response was $22 \%(95 \% \mathrm{CI}=13-34 \%)$ per patient-year. The cumulative incidence of loss of response was $38 \%(95 \% \mathrm{CI}=23-55 \%): 18 \%$ at 1 year and $37 \%$ at 2 years of follow-up (Figure 4). In the univariate analysis, none of the variables studied were associated with the probability of loss of efficacy (gender, age at diagnosis, type of IBD, reasons to withdraw the second antiTNF, combo therapy, smoking history and extraintestinal manifestations). The multivariate analysis could not be performed due to de small sample size (39 patients). 
Seven patients (11\%) had adverse events. Of these, 3 patients had infection (1 herpes, 1 bronchitis and 1 perianal abscesses), 2 patients had psoriasis, 1 paraesthesias in lower limbs, and 1 delayed hypersensitivity reaction. Only one patient (the patient with perianal abscesses) discontinued the therapy due to the adverse event. 


\section{DISCUSSION}

To the best of our knowledge, this is the largest cohort of IBD patients $(1,122$ patients) in whom the strategy of switching to a second anti-TNF after intolerance, primary failure, or secondary failure to the first one has been evaluated. Moreover, the present study is one of the few studies which assess the sequential administration of a third anti-TNF after failure of the second one.

In IBD patients, anti-TNF agents are efficacious in inducing and maintaining disease remission. Nevertheless, a group of patients have no response or have a partial response to these agents. Furthermore, patients who initially respond may lose efficacy over time or develop intolerance, which sometimes leads to stopping treatment. In these possible scenarios, switching from one anti-TNF agent to another could represent an option ${ }^{13}$.

In our study, $45 \%$ of the patients achieved remission with the second anti-TNF in the short-term. These results are in agreement with other studies ${ }^{2,14-18}$. We also found that nearly half of patients diagnosed with CD achieved remission with the second anti-TNF. In CD patients, several studies had proven that a second anti-TNF therapy is efficacious in those patients who are intolerant or lose response to the prior anti-TNF drug ${ }^{13,16,18-21}$. However, only few studies with small sample sizes have analyzed the effectiveness of the strategy of the sequential use of a second anti-TNF if the reason for withdrawal of the first drug was primary

failure $^{14-16,21-27}$. The rates of remission in these studies were highly variable, ranging from $11 \%$ to $60 \%$ at one year.

On the other hand, in UC patients, only few studies with small number of patients and heterogeneous designs had evaluated the effectiveness of a second anti-TNF drug, with reported rates of remission from $0 \%$ to $50 \%^{3,7-9}$. We found that, in the short-term, UC patients had an overall remission rate of $41 \%$. Moreover, the remission rate in CD patients 
was similar to UC patients in the short-term; however, in the long-term, patients with UC had a higher probability of losing efficacy than CD patients. Sandborn et al. assessed the efficacy of ADA after IFX in 98 UC patients. At one year, the rate of remission with the second antiTNF was $10 \%$. However, the reason for withdrawal the first anti-TNF withdrawal in these patients was not reported ${ }^{8}$. Nonetheless, in UC patients, remission rate to a second anti-TNF seems to be lower than remission rate in CD patients. Therefore, further studies are necessary in UC patients, in order to investigate the efficacy of a second anti-TNF drug in those patients with intolerance, primary failure or secondary failure to the first drug.

In the present study, the probability of achieving remission at short-term was associated with the reason for discontinuing the first anti-TNF. In fact, patients who discontinued the first anti-TNF due to primary or secondary failure had a lower probability of achieving remission than those whose reason for switching was intolerance. According to our results, a very recent study evaluated the efficacy of ADA and vedolizumab in $161 \mathrm{UC}$ patients who were previously treated with IFX. The authors reported that the efficacy of ADA was similar to vedolizumab in patients who were IFX intolerants. However, vedolizumab was more effective than ADA in IFX secondary failures ${ }^{28}$. These results are similar than those reported in a recent meta-analysis, in which the remission rate in the short-term was higher when the reason for switching was intolerance $(50 \%)$ in comparison with primary $(18 \%)$ or secondary failure $(41 \%)^{2}$.

According to our results, of patients who switched to a second anti-TNF due to primary failure to the first one, approximately $40 \%$ achieved remission. This remission rate after primary failure was slightly lower than in other studies ${ }^{14,} 18,25$. This finding could be explained by the small sample sizes of the previous studies. Since primary failure is considered a class effect phenomenon, the similar structure and function shared by the antiTNF drugs might lead to assume that if a patient was a non-responder to the first anti-TNF 
will not respond to the second ${ }^{29,30}$. However, our results indicate that remission may still be achieved with a second anti-TNF in approximately $50 \%$ of the patients after primary nonresponse to a prior drug. In according with our results, switching to another anti-TNF after primary failure may still be a valid therapeutic option in IBD; especially considering that the therapeutic armamentarium of IBD is still limited.

We found that in patients in remission with the second anti-TNF, the incidence of loss of response was relatively high: $19 \%$ per patient-year, with a cumulative incidence of loss of response of $45 \%$ in 19 months (IQR 8-40) of follow-up. These results are similar to other smaller studies $^{14,31-33}$.

In the multivariate analysis, we found that in patients who switched to a second anti-TNF, the long-term effectiveness was associated with the reason for switching the first drug. In fact, the probability of remission of the patients treated with a second anti-TNF was lower in those patients who switched due to primary or secondary failure to the first one, in comparison with those patients who switched due to intolerance. These results are consistent with a recent meta-analysis by our group that included 35 studies and evaluated the efficacy of a second anti-TNF when the previous has failed. In this study, the patients who discontinued the first anti-TNF due to intolerance had higher rates of remission (61\%) than those who switched to a second anti-TNF due to primary $(30 \%)$ or secondary failure $(45 \%)^{2}$. Only one randomized trial has specifically evaluated the efficacy of a second-line anti-TNF agent in patients diagnosed with $\mathrm{CD}$ who were intolerant of or lost their response to a first anti-TNF drug (IFX) ${ }^{19}$. In this study, ADA proved to be superior to placebo for inducing remission and response. The reason for discontinuing IFX (intolerance vs. secondary failure) had no impact in clinical improvement; however, the primary endpoint of this study was remission in week 4 (short-term).

Surprisingly, in patients who switched to a second anti-TNF and were on combination 
therapy with immunomodulators, the probability of remission was lower. An explanation for this fact could be that patients who were on combo therapy probably had a more aggressive disease than those on monotherapy. In fact, we found that in patients on combo therapy the duration of the disease until the start of both the first and second anti-TNF was shorter than in patients on monotherapy, which suggests that these patients probably had a more aggressive disease. Nonetheless, in accordance with the results of a recent meta-analysis, combo therapy after starting anti-TNF therapy is no more effective than anti-TNF in monotherapy (IFX or ADA) in inducing or maintaining remission in patients who have been previously exposed to anti-TNF ${ }^{34}$

One-year after switching, $77 \%$ out of $45 \%$ IBD patients who achieved remission with the second anti-TNF maintained remission. This finding is similar to other study which included 118 patients diagnosed with $\mathrm{CD}$ who switched to a second anti-TNF after failure of the previous anti-TNF ${ }^{14}$. Therefore, a considerable number of patients who switch to a second anti-TNF will be in remission in the first year; however, a high proportion of these patients will lose efficacy along time. Accordingly, the sequential use of a second anti-TNF is an option that should be considered, especially in those patients in whom the reason for switching was intolerance.

In the present study, we found a higher risk of loss of efficacy in UC patients than in CD patients. This finding was reported by a meta-analysis which included 6 studies in UC patients who had primary or secondary failure to the first anti-TNF and switched to a second drug. The overall remission rates in these studies were lower than the reported in CD patients $^{2}$. Nevertheless, more prospective studies are needed to investigate the effectiveness of a second anti-TNF agent in UC patients in whom the first drug has failed.

In IBD patients, dose-escalation of the anti-TNF drug is a common and effective strategy in those patients who lose their response to anti-TNF drugs ${ }^{1}$. According to our results, 
approximately $20 \%$ of the patients who achieved remission with the second anti-TNF escalated the dose due to loss of response. Of these, 70\% regained remission. This elevated rate of remission could be explained by the fact that all the patients escalated the dose due to secondary failure. Almost half of the cohort of patients who switched to a second anti-TNF agent and who escalated the dose of the drug achieved remission. Of these, almost two-thirds of the patients who escalated the dose due to partial response and half of the patients who did it due to secondary failure regained remission. However, none of the patients who escalated the dose due to non-response regained remission. These results are similar to those of another study ${ }^{14}$. Our findings suggest that the escalation of the dose of the anti-TNF drug in patients who lose their response to a second anti-TNF may also be considered a valid therapeutic strategy. Moreover, according to our results, an important number of patients with partial response to a second anti-TNF could benefit from dose escalation of the drug. None of the non-responder patients who escalated the dose of the second anti-TNF achieved remission. Nevertheless, the number of these patients was small. For this reason, there are not enough data to recommend the escalation of the dose of the anti-TNF in patients who do not respond to the second anti-TNF.

The sequential use of anti-TNF drugs is potentially risky since the second agent may worsen the immunosuppression induced by the first agent ${ }^{35}$. Of the total of patients who switched to a second drug, $15 \%$ had adverse events. Two-thirds of them $(10 \%$ of the total cohort) discontinued the drug due to adverse events. In a systematic review of the use of ADA after IFX failure in CD patients, adverse events were reported in $13 \%$ to $69 \%$ of the patients, and the severity of the majority these effects were mild to moderate, with a rate of discontinuation ranging from $0 \%$ to $14 \%^{32}$.

In clinical practice, it is not exceptional the switch to a third anti-TNF drug; however, data are limited. ${ }^{35}$. In our study, we report the largest cohort of patients who switched to a third 
anti-TNF after intolerance or failure to the second one. In the short-term, of the 71 patients that were included, approximately 50\% achieved remission while two-thirds of them were in remission at 24 months. Two studies have assessed the remission rate of a third anti-TNF. Allez et al. studied 67 patients diagnosed with CD that were treated with a third anti-TNF due to intolerance or failure to the second drug. In week six, $61 \%$ of the patients had clinical response while $51 \%$ of the patients had clinical response at week $20{ }^{36}$. Silva et al. studied 63 IBD patients who received a third anti-TNF after intolerance or loss of response. Remission was achieved in $36 \%$ of these patients, and over $50 \%$ of the patients who achieved remission remained on the third anti-TNF after one year ${ }^{37}$ In our study, the probability of maintaining remission after the third anti-TNF was higher than Silva et. al. study. However, the long-term benefit of a third anti-TNF agent is still unknown.

We found that $11 \%$ of patients had adverse events, although only in one of them the drug had to be withdrawn. However, the use of a third anti-TNF raises additional safety concerns. Allez et al. reported that 14 patients had to discontinue the third anti-TNF due to severe adverse events. Also, two deaths were reported by the authors ${ }^{36}$. Nonetheless, there were no deaths reported in the study of Silva et. al. during the follow-up period ${ }^{37}$. Although the efficacy of switching to a third anti-TNF seems to be relatively favorable, this strategy cannot be generally recommended because of the risk of severe adverse events. Especially because there are available for the treatment of IBD other recently approved medical options (e.g. vedolizumab, ustekinumab) that are effective and safe.

The present study has several limitations. First, information about anti-TNF trough levels, anti-drug antibodies and endoscopic activity was not available. Nonetheless, due to the fact that this is a retrospective study the investigators made an effort in order to obtain information on all components of clinical indices. Second, since this was a real-life study, the treating physicians decided to discontinue one anti-TNF and switch to another based on their criteria. 
Third, the number of patients who switched to a third anti-TNF was small, and the follow-up period of these patients was relatively short. However, the number of patients in which this strategy was evaluated is one of the largest that has been published.

One of the main strengths of this study is that it reflects real-life clinical practice. Moreover, this is the largest cohort of IBD patients in whom the strategy of switching to a second anti-TNF after intolerance, primary failure or secondary failure to the first one has been evaluated. In addition, this is the largest study in which the strategy of the sequential use of a third anti-TNF has been evaluated.

In conclusion, our results suggest that the sequential use of a second anti-TNF is efficacious in IBD patients after intolerance, primary failure or secondary failure to a first anti-TNF. In any case, a high proportion of the patients who achieve remission with a second anti-TNF lose response afterward. Combination therapy with immunomodulators and type of IBD are predictors of loss of response to a second anti-TNF. Of the patients treated with a third anti-TNF, two-thirds achieved remission with the drug; nonetheless, a significant number of these patients experienced loss of efficacy over time. Finally, the sequential use of a second anti-TNF is safe; although switching to a third anti-TNF seems to be relatively safe, this last strategy cannot be generally recommended in clinical practice. 


\section{REFERENCES}

1 Gisbert JP, Panes J. Loss of response and requirement of infliximab dose intensification in Crohn's disease: a review. The American journal of gastroenterology. 2009;104(3):760-767.

2 Gisbert JP, Marin AC, McNicholl AG, Chaparro M. Systematic review with metaanalysis: the efficacy of a second anti-TNF in patients with inflammatory bowel disease whose previous anti-TNF treatment has failed. Alimentary pharmacology \& therapeutics. 2015;41(7):613-623.

3 Afif W, Leighton JA, Hanauer SB, et al. Open-label study of adalimumab in patients with ulcerative colitis including those with prior loss of response or intolerance to infliximab. Inflammatory bowel diseases. 2009;15(9):1302-1307.

4 Barreiro-de Acosta M, Garcia-Bosch O, Gordillo J, et al. Efficacy of adalimumab rescue therapy in patients with chronic refractory pouchitis previously treated with infliximab: a case series. European journal of gastroenterology \& hepatology. 2012;24(7):756-758.

5 Garcia-Bosch O, Gisbert JP, Canas-Ventura A, et al. Observational study on the efficacy of adalimumab for the treatment of ulcerative colitis and predictors of outcome. Journal of Crohn's \& colitis. 2013;7(9):717-722.

6 Oussalah A, Laclotte C, Chevaux JB, et al. Long-term outcome of adalimumab therapy for ulcerative colitis with intolerance or lost response to infliximab: a single-centre experience. Alimentary pharmacology \& therapeutics. 2008;28(8):966-972.

7 Peyrin-Biroulet L, Laclotte C, Roblin X, Bigard MA. Adalimumab induction therapy for ulcerative colitis with intolerance or lost response to infliximab: an open-label study. World journal of gastroenterology : WJG. 2007;13(16):2328-2332.

8 Sandborn WJ, van Assche G, Reinisch W, et al. Adalimumab induces and maintains clinical remission in patients with moderate-to-severe ulcerative colitis. Gastroenterology. 
2012;142(2):257-265 e251-253.

9 Taxonera C, Estelles J, Fernandez-Blanco I, et al. Adalimumab induction and maintenance therapy for patients with ulcerative colitis previously treated with infliximab. Alimentary pharmacology \& therapeutics. 2011;33(3):340-348.

10 Chaparro M, Martinez-Montiel P, Van Domselaar M, et al. Intensification of infliximab therapy in Crohn's disease: efficacy and safety. Journal of Crohn's \& colitis. 2012;6(1):62-67.

11 Harvey RF, Bradshaw JM. A simple index of Crohn's-disease activity. Lancet. 1980;1(8167):514.

12 D'Haens G, Feagan B, Colombel JF, et al. Challenges to the design, execution, and analysis of randomized controlled trials for inflammatory bowel disease. Gastroenterology. 2012;143(6):1461-1469.

13 Chaparro M, Andreu M, Barreiro-de Acosta M, et al. Effectiveness of infliximab after adalimumab failure in Crohn's disease. World journal of gastroenterology : WJG. 2012;18(37):5219-5224.

14 C RGM, Chaparro M, Mesonero F, et al. Effectiveness of anti-TNFalpha drugs in patients with Crohn's disease who do not achieve remission with their first anti-TNFalpha agent. Digestive and liver disease : official journal of the Italian Society of Gastroenterology and the Italian Association for the Study of the Liver. 2016;48(6):613-619.

15 Ho GT, Mowat A, Potts L, et al. Efficacy and complications of adalimumab treatment for medically-refractory Crohn's disease: analysis of nationwide experience in Scotland (2004-2008). Alimentary pharmacology \& therapeutics. 2009;29(5):527-534.

16 Lofberg R, Louis EV, Reinisch W, et al. Adalimumab produces clinical remission and reduces extraintestinal manifestations in Crohn's disease: results from CARE. Inflammatory bowel diseases. 2012;18(1):1-9. 
17 Sprakes MB, Hamlin PJ, Warren L, Greer D, Ford AC. Adalimumab as second line anti-tumour necrosis factor alpha therapy for Crohn's disease: A single centre experience. Journal of Crohn's \& colitis. 2011;5(4):324-331.

18 Swaminath A, Ullman T, Rosen M, Mayer L, Lichtiger S, Abreu MT. Early clinical experience with adalimumab in treatment of inflammatory bowel disease with infliximabtreated and naive patients. Alimentary pharmacology \& therapeutics. 2009;29(3):273-278.

19 Sandborn WJ, Rutgeerts P, Enns R, et al. Adalimumab induction therapy for Crohn disease previously treated with infliximab: a randomized trial. Annals of internal medicine. 2007;146(12):829-838.

20 Sandborn WJ, Abreu MT, D'Haens G, et al. Certolizumab pegol in patients with moderate to severe Crohn's disease and secondary failure to infliximab. Clinical gastroenterology and hepatology : the official clinical practice journal of the American Gastroenterological Association. 2010;8(8):688-695 e682.

21 Swoger JM, Loftus EV, Jr., Tremaine WJ, et al. Adalimumab for Crohn's disease in clinical practice at Mayo clinic: the first 118 patients. Inflammatory bowel diseases. 2010;16(11):1912-1921.

22 Barthel HR, Gille T, Halbsguth A, Kramer M. Successful treatment with adalimumab in infliximab-resistant Crohn's disease. Journal of gastroenterology and hepatology. 2005;20(9):1464-1465.

23 Cordero Ruiz P, Castro Marquez C, Mendez Rufian V, et al. Efficacy of adalimumab in patients with Crohn's disease and failure to infliximab therapy: a clinical series. Revista espanola de enfermedades digestivas : organo oficial de la Sociedad Espanola de Patologia Digestiva. 2011;103(6):294-298.

24 Lichtiger S, Binion DG, Wolf DC, et al. The CHOICE trial: adalimumab demonstrates safety, fistula healing, improved quality of life and increased work productivity in patients 
with Crohn's disease who failed prior infliximab therapy. Alimentary pharmacology \& therapeutics. 2010;32(10):1228-1239.

25 Ho GT, Smith L, Aitken S, et al. The use of adalimumab in the management of refractory Crohn's disease. Alimentary pharmacology \& therapeutics. 2008;27(4):308-315.

26 Panaccione R, Loftus EV, Jr., Binion D, et al. Efficacy and safety of adalimumab in Canadian patients with moderate to severe Crohn's disease: results of the Adalimumab in Canadian SubjeCts with ModErate to Severe Crohn's DiseaSe (ACCESS) trial. Canadian journal of gastroenterology = Journal canadien de gastroenterologie. 2011;25(8):419-425.

27 Seiderer J, Brand S, Dambacher J, et al. Adalimumab in patients with Crohn's disease-safety and efficacy in an open-label single centre study. Alimentary pharmacology \& therapeutics. 2007;25(7):787-796.

28 Favale A, Onali S, Caprioli F, et al. Comparative Efficacy of Vedolizumab and Adalimumab in Ulcerative Colitis Patients Previously Treated With Infliximab. Inflammatory bowel diseases. 2019.

29 Ben-Horin S, Kopylov U, Chowers Y. Optimizing anti-TNF treatments in inflammatory bowel disease. Autoimmunity reviews. 2014;13(1):24-30.

30 Dassopoulos T. When the love is lost: adalimumab for patients with an attenuated response to infliximab. Inflammatory bowel diseases. 2005;11(10):948-949.

31 Ma C, Panaccione R, Heitman SJ, Devlin SM, Ghosh S, Kaplan GG. Systematic review: the short-term and long-term efficacy of adalimumab following discontinuation of infliximab. Alimentary pharmacology \& therapeutics. 2009;30(10):977-986.

32 Da W, Zhu J, Wang L, Lu Y. Adalimumab for Crohn's disease after infliximab treatment failure: a systematic review. European journal of gastroenterology \& hepatology. $2013 ; 25(8): 885-891$.

33 Chaparro M, Panes J, Garcia V, et al. Long-term durability of response to adalimumab 
in Crohn's disease. Inflammatory bowel diseases. 2012;18(4):685-690.

34 Jones JL, Kaplan GG, Peyrin-Biroulet L, et al. Effects of Concomitant Immunomodulator Therapy on Efficacy and Safety of Anti-Tumor Necrosis Factor Therapy for Crohn's Disease: A Meta-analysis of Placebo-controlled Trials. Clinical gastroenterology and hepatology : the official clinical practice journal of the American Gastroenterological Association. 2015;13(13):2233-2240 e2231-2232; quiz e2177-2238.

35 Gisbert JP, Chaparro M. Use of a third anti-TNF after failure of two previous antiTNFs in patients with inflammatory bowel disease: is it worth it? Scandinavian journal of gastroenterology. 2015;50(4):379-386.

36 Allez M, Vermeire S, Mozziconacci N, et al. The efficacy and safety of a third antiTNF monoclonal antibody in Crohn's disease after failure of two other anti-TNF antibodies. Alimentary pharmacology \& therapeutics. 2010;31(1):92-101.

37 de Silva PS, Nguyen DD, Sauk J, Korzenik J, Yajnik V, Ananthakrishnan AN. Longterm outcome of a third anti-TNF monoclonal antibody after the failure of two prior antiTNFs in inflammatory bowel disease. Alimentary pharmacology \& therapeutics. 2012;36(5):459-466. 
Table 1. Baseline characteristics of the patients.

\begin{tabular}{|c|c|}
\hline Variables & Total \\
\hline $\begin{array}{l}\text { Type of disease, n (\%) } \\
\text { Crohn's disease } \\
\text { Ulcerative colitis }\end{array}$ & $\begin{array}{l}1.122(100) \\
822(73.3) \\
300(26.7)\end{array}$ \\
\hline Male, $\mathrm{n}(\%)$ & $563(50.2)$ \\
\hline Mean age at diagnosis, years (range) & $30(5-79)$ \\
\hline $\begin{array}{l}\text { Median time from diagnosis to the start of the } \\
\text { anti-TNF therapy, months (IQR) } \\
\text { First anti-TNF } \\
\text { Second anti-TNF } \\
\text { Third anti-TNF }\end{array}$ & $\begin{array}{l}44(12-120) \\
65(28-141) \\
76(48-134) \\
\end{array}$ \\
\hline Extra-intestinal manifestations, n (\%) & $417(37.2)$ \\
\hline Smoking history, $\mathrm{n}(\%)$ & $383(34.1)$ \\
\hline History of abdominal surgery, $\mathrm{n}(\%)$ & $341(56.9)$ \\
\hline $\begin{array}{l}\text { Type of first anti-TNF therapy, n (\%) } \\
\text { Infliximab } \\
\text { Adalimumab } \\
\text { Certolizumab } \\
\text { Golimumab }\end{array}$ & $\begin{array}{l}752(67) \\
348(31) \\
1(0.1) \\
21(1.9)\end{array}$ \\
\hline $\begin{array}{l}\text { Type of second anti-TNF therapy, n (\%) } \\
\text { Infliximab } \\
\text { Adalimumab } \\
\text { Certolizumab } \\
\text { Golimumab }\end{array}$ & $\begin{array}{l}354(31.6) \\
728(64.9) \\
14(1.2) \\
26(2.3)\end{array}$ \\
\hline $\begin{array}{l}\text { Type of third anti-TNF therapy, } \mathrm{n}(\%) \\
\text { Infliximab } \\
\text { Adalimumab } \\
\text { Certolizumab } \\
\text { Golimumab }\end{array}$ & $\begin{array}{l}3(4.2) \\
8(11.3) \\
36(50.7) \\
24(33.8)\end{array}$ \\
\hline $\begin{array}{l}\text { Concomitant IMMs, n (\%) } \\
\text { First anti-TNF } \\
\text { Second anti-TNF } \\
\text { Third anti-TNF }\end{array}$ & $\begin{array}{l}694(62) \\
370(33) \\
16(22.5)\end{array}$ \\
\hline $\begin{array}{l}\text { Reasons for the first anti-TNF discontinuation, } \\
\mathrm{n}(\%) \\
\text { Non-response } \\
\text { Partial response } \\
\text { Loss of efficacy } \\
\text { Adverse events }\end{array}$ & $\begin{array}{l}124(11.1) \\
117(10.4) \\
573(51.1) \\
308(27.5)\end{array}$ \\
\hline $\begin{array}{l}\text { Montreal location at Crohn's disease diagnosis, } \\
\text { n (\%) } \\
\text { L1 (ileal) } \\
\text { L2 (colonic) } \\
\text { L3 (ileocolonic) } \\
\quad \text { L4 (upper gastrointestinal tract) }\end{array}$ & $\begin{array}{l}281(34.2) \\
155(18.9) \\
385(46.8) \\
1(0.1)\end{array}$ \\
\hline
\end{tabular}




\begin{tabular}{|c|l|}
\hline Montreal behavior at Crohn's disease & \\
diagnosis, $\mathrm{n}(\%)$ & $441(53.6)$ \\
B1 (inflammatory) & $199(24.2)$ \\
B2 (stricturing) & $182(22.1)$ \\
B3 (penetrating) & $358(43.7)$ \\
\hline Perianal disease, $\mathrm{n}(\%)$ & $15(5)$ \\
\hline Ulcerative colitis extension, n (\%) & $109(36.3)$ \\
Proctitis & $175(58.3)$ \\
Left side colitis & $1(0.3)$ \\
Extensive colitis & \\
Unknown & \\
\hline
\end{tabular}

IMMs: immunomodulators; IQR: interquartile range. 
Table 2. Multivariate analysis of factors associated with the probability of achieving remission with the second anti-TNF.

\begin{tabular}{|l|c|c|c|}
\hline Factors & OR & $\mathbf{9 5 \% ~ C I ~}$ & P-value \\
\hline Concomitant IMMs & 0.5 & $0.4-0.7$ & $<0.0001$ \\
\hline $\begin{array}{l}\text { To withdraw the first anti-TNF } \\
\text { due to a primary failure (vs. } \\
\text { intolerance) }\end{array}$ & 0.6 & $0.4-0.9$ & 0.007 \\
\hline $\begin{array}{l}\text { To withdraw the first anti-TNF } \\
\text { due to secondary failure (vs. } \\
\text { intolerance) }\end{array}$ & 0.6 & $0.5-0.9$ & 0.003 \\
\hline Gender & 0.84 & $0.7-1.1$ & 0.1 \\
\hline Age at diagnosis & 0.9 & $0.9-1$ & 0.1 \\
\hline Smoking history & 1.1 & $0.9-1.5$ & 0.4 \\
\hline Type of IBD (UC vs. CD) & 0.9 & $0.7-1.3$ & 0.5 \\
\hline Extraintestinal manifestations & 0.9 & $0.7-1.2$ & 0.5 \\
\hline Duration of disease & 1 & $0.99-1.001$ & 0.9 \\
\hline
\end{tabular}

OR: odds ratio; IMMs: immunomodulators; CI: confidence interval; IBD: inflammatory bowel disease; UC: ulcerative colitis; CD: Crohn's disease. 
Table 3. Adverse events with the second anti-TNF drug

\begin{tabular}{|l|c|}
\hline Adverse event & N (\%) \\
\hline Infusion reaction & $45(26.4)$ \\
\hline Infections & $45(26.4)$ \\
\hline Delayed hypersensitivity reaction & $26(15.3)$ \\
\hline Toxicodermia & $17(10)$ \\
\hline Drug-induced psoriasis & $15(8.8)$ \\
\hline Drug-induced lupus & $8(4.7)$ \\
\hline Headache & $5(3)$ \\
\hline Malignancy & $4(2.4)$ \\
\hline Thrombocytopenia & $3(1.8)$ \\
\hline Anaphylaxis & $1(0.6)$ \\
\hline Myelitis & $1(0.6)$ \\
\hline
\end{tabular}


Table 4. Baseline characteristics of the patients who switched to a third anti-TNF.

\begin{tabular}{|l|l|}
\hline Variables & Total \\
\hline Type of disease, $\mathrm{n}(\%)$ & $71(100)$ \\
-Crohn's disease & $45(63.4)$ \\
-Ulcerative colitis & $26(36.6)$ \\
\hline Male, $\mathrm{n}(\%)$ & $39(54.9)$ \\
\hline Mean age at diagnosis, years (range) & $32(11-75)$ \\
\hline Extra-intestinal manifestations, $\mathrm{n}(\%)$ & $29(40.8)$ \\
\hline Smoking history, $\mathrm{n}(\%)$ & $22(34.9)$ \\
\hline History of abdominal surgery, $\mathrm{n}(\%)$ & $20(28.2)$ \\
\hline Reasons for the second anti-TNF & \\
discontinuation, $\mathrm{n}(\%)$ & $32(45.1)$ \\
-Primary failure & $28(39.4)$ \\
-Secondary failure & $11(15.5)$ \\
\hline -Adverse events & \\
\hline Montreal location at Crohn's disease diagnosis, & $14(31.1)$ \\
$\mathrm{n}$ (\%) & $9(20)$ \\
-L1 (ileal) & $22(48.9)$ \\
-L2 (colonic) & \\
-L3 (ileocolonic) & $20(44.4)$ \\
\hline Montreal behaviour at Crohn's disease & $15(33.3)$ \\
diagnosis, $\mathrm{n}(\%)$ & $10(22.2)$ \\
\hline -B1 (inflammatory) & $19(42.2)$ \\
-B2 (stricturing) & 0 \\
-B3 (penetrating) & $14(53.8)$ \\
\hline Perianal disease, $\mathrm{n}(\%)$ & \\
\hline Ulcerative colitis extension, $\mathrm{n}(\%)$ & \\
-Proctitis & \\
-Left side colitis & \\
-Extensive colitis & \\
\hline
\end{tabular}




\section{Figure legends:}

Figure 1. Kaplan-Meier curve of long-term remission after treatment with a second anti-TNF.

Figure 2. Kaplan-Meier analysis of long-term remission after treatment with a second antiTNF in Crohn's disease vs. ulcerative colitis patients.

Figure 3. Kaplan-Meier analysis of long-term remission after treatment with a second antiTNF in patients treated with immunomodulators vs. untreated patients.

Figure 4. Kaplan-Meier curve of long-term remission after treatment with a third anti-TNF.

Figure 5. Flow chart of the patients who switched to a second and a third anti-TNF. 